\title{
Imperative Instruction for Pressurized Metered-Dose Inhalers: Provider Perspectives
}

\author{
Daniel C Schmitz, Rebecca A Ivancie, Kyung E Rhee, Heather C Pierce, Alicia O Cantu, and \\ Erin S Fisher
}

\begin{abstract}
BACKGROUND: Reports show that many patients do not use their pressurized metered-dose inhalers (pMDIs) effectively. The National Heart, Lung, and Blood Institute recommends that health-care providers educate and assess patients' pMDI technique at each opportunity. However, limited data exist regarding how often pediatric primary care providers perform assessments and which methods they use. We sought to (1) identify instructional methods used to teach pMDI use, (2) describe how pMDI use is reassessed at follow-up visits, and (3) describe primary care provider attitudes and barriers to in-office pMDI instruction. METHODS: A 34-item electronic survey was distributed from August to December 2016 via E-mail to local pediatric primary care providers. Descriptive statistics were used for analysis. RESULTS: Sixty two of $\mathbf{2 2 3}$ potential primary care providers $(28 \%)$ responded. Physicians and nurse practitioners were identified most often as the providers of pMDI education (53\%). When first prescribing a pMDI, only $10 \%$ reported having the patient practice inhaler use in the office and receive feedback. Only 19\% "always" reassessed the technique, even for patients with poorly controlled asthma. Among those who reassessed the technique, most $(76 \%)$ did so verbally, and only $42 \%$ asked the patients to demonstrate pMDI use. Only 32\% reported that typical patient education in their setting was adequate to ensure proper pMDI use. Commonly cited barriers included time (84\%) and access to demo pMDIs (67\%). Provider solutions included video tutorials and access to demo inhalers. CONCLUSIONS: Many pediatric primary care providers did not demonstrate or have patients practice pMDI use when teaching or assessing pMDI technique, and the reassessment rate was low even for patients with poorly controlled asthma. Identifying and training a consistent pMDI educator and obtaining demo pMDIs may abate some barriers. Respiratory therapists could appropriately fulfill this educator role. Brief, repeated pMDI practice for motor learning could promote more stable pMDI mastery. Key words: asthma; metered-dose inhaler; education; reassessment; technique; motor learning. [Respir Care 2019;64(3):292-298. (C) 2019 Daedalus Enterprises]
\end{abstract}

\section{Introduction}

Asthma is a common and potentially debilitating chronic disease that affects $>6.2$ million children in the United

Mr Schmitz is affiliated with the University of California San Diego School of Medicine, La Jolla, California. Dr Ivancie is affiliated with the Division of Hospital Medicine, Department of Pediatrics, Stanford University School of Medicine, Palo Alto, California. Dr Rhee is affiliated with the Division of Academic General Pediatrics, Child Development, and Community Health, Department of Pediatrics, University of California San Diego School of Medicine, La Jolla, California. Drs Pierce, Cantu, and Fisher are affiliated with the Rady Children's Hospital and Health Centers, Division of Hospital Medicine, Department of Pediatrics,
States. ${ }^{1}$ In many cases the disease is managed by using inhaled medications dispensed via common devices such as pressurized metered-dose inhalers (pMDIs). The proper use of such devices is essential to effective medication administration because up to $80 \%$ of medication may be

University of California San Diego School of Medicine, San Diego, California.

Mr Schmitz presented part of these data at the American Academy of Pediatrics National Convention and Exhibition, held September 16-19, 2017, in Chicago, Illinois.

The authors have disclosed no conflicts of interest. 
lost due to improper technique. ${ }^{2}$ Studies have shown that $28 \%$ to $68 \%$ of subjects of all ages did not use their inhaler effectively enough to benefit from the prescribed medication. ${ }^{3,4}$ In addition, poor inhalation technique has been associated with excess use of health-care resources, including hospitalizations, emergency department visits, and additional courses of oral corticosteroids. ${ }^{5}$ Establishing and sustaining proper inhaler technique, therefore, represents a low-cost intervention vital to successful long-term asthma management.

A variety of educational techniques have been shown to improve inhaler technique in the short term. ${ }^{6}$ However, skills in inhaler use deteriorate over time, ${ }^{6,7}$ which indicates that a single instruction session may not be sufficient for patients to maintain proper technique. Previous studies have shown that repeating instruction sessions, demonstrating inhaler technique to patients, and having patients return the demonstration were all associated with higher rates of proper technique at follow-up visits..$^{5,8-11}$ These aspects of effective inhaler instruction were incorporated into the National Heart, Lung, and Blood Institute (NHLBI) guidelines. ${ }^{12}$ These guidelines recommend that providers teach and reinforce inhaler skills at every visit by demonstrating inhaler technique, having the patient return the demonstration, and offering feedback.

Analysis of existing data, however, indicates that healthcare providers do not consistently follow the NHLBI guidelines $^{12}$ for inhaler instruction. In one study of 5 general pediatric clinics in North Carolina, providers demonstrated inhaler technique to just $3.8 \%$ of children who used pMDIs and asked only $5.4 \%$ of these children to demonstrate their inhaler technique during the clinic visit. ${ }^{13}$ Many of the children in this study were subsequently found to make several errors in inhaled medication administration. ${ }^{13}$ Similarly, in a recent survey of pediatric out-patient providers in New York, only $5.3 \%$ of providers reported demonstrating the pMDI technique at every visit and just $13.3 \%$ reported assessing patient technique at every visit. ${ }^{14}$ When asked to identify barriers to demonstrating and assessing pMDI technique, providers cited a lack of access to pMDI devices, time, and knowledge of proper steps in pMDI use, and patient or parent disinterest in learning or in demonstrating the proper technique. ${ }^{14}$ Together, results of these studies $^{13,14}$ indicate that, for a variety of reasons, many

Supplementary material related to this paper is available at http:// www.rcjournal.com.

Correspondence: Erin S Fisher MD, Rady Children's Hospital and Health Centers, Division of Hospital Medicine, Department of Pediatrics, University of California San Diego School of Medicine, 7960 Birmingham Drive, San Diego, CA 92123. E-mail: estucky@ rchsd.org.

DOI: $10.4187 /$ respcare.06302

\section{QUICK LOOK}

\section{Current knowledge}

Proper pressurized metered-dose inhaler (pMDI) use is essential for management of asthma symptoms, but there are reports that many patients do not use them effectively. The National Heart, Lung, and Blood Institute recommends that health-care providers educate and assess patients' pMDI technique at each opportunity. There are limited data about how often pediatric primary care providers perform assessments and the methods they use.

\section{What this paper contributes to our knowledge}

Many pediatric primary care providers did not demonstrate or have patients practice pMDI use when teaching or assessing pMDI technique. The reassessment rate was low, even for patients with poorly controlled asthma. Commonly cited challenges included time, access to demo pMDIs, perceived patient disinterest, and language barriers.

providers do not implement NHLBI guidelines ${ }^{12}$ regarding pMDI instruction in their practices.

These studies were limited in scope, however, because the former did not address reasons for suboptimal instruction, whereas the latter focused on providers in pediatric primary care settings that serve as residency training sites in an urban low-income community. ${ }^{13,14}$ The goal of our study was to further examine how pMDI instruction takes place in a wider range of out-patient settings and what challenges remain to effective instruction. We aimed to describe instructional methods used to teach pMDI use and identify who performs this teaching, describe how pMDI use is reassessed at follow-up visits, and describe provider attitudes and self-reported barriers with regard to in-office pMDI instruction. We hypothesized that, in these out-patient settings with differing care delivery models, there would not be a single common instructional method used for pMDI instruction but that methods would include verbal instructions and the use of printed material, that providers would report not assessing and providing pMDI instruction as recommended by the NHLBI ${ }^{12}$ at follow-up visits, and that providers would report varied barriers to delivering pMDI instruction.

\section{Methods}

This cross-sectional survey-based study was conducted in San Diego County between August and December 2016. Several out-patient pediatric practice types, including an 


\section{pMdi Instruction in Pediatric Primary Care}

academic center (1 group), large pediatric group practices (5 groups), federally qualified health centers (1 group), and private practices (6 groups), were included. Leaders within each of these groups were asked to distribute the survey link via E-mail to pediatric physicians, nurse practitioners, and physician assistants $(N=223)$ within their group by using the E-mail addresses in active use for their respective providers. The survey was distributed 4 times within a 4-month period to increase response rates. No incentive was provided for participation. This study (Approval Project 160646) was approved by the University of California San Diego Human Research Protections Program.

\section{Survey}

A 34-item survey was developed to assess pMDI instructional practices and provider attitudes, and perceived barriers to pMDI instruction. Because there were no previously validated instruments on this topic, questions were generated based on peer-reviewed literature ${ }^{13,14}$ and clinical experience. Questions were reviewed and revised by us. Items included multiple choice, Likert scale, and freeresponse formats. The survey included questions regarding the following: type of medication delivery device prescribed and prescribing habits, form and frequency of pMDI education and reassessment, attitudes toward inhaler education, perceived barriers to inhaler education, personal educational experiences regarding how to use an inhaler and how to teach these steps to patients, confidence in and perceived effectiveness of their instruction, and methods or resources that the providers thought would be most effective to teach proper technique (for survey questions, see the supplementary materials at http://www.rcjournal. com). The survey was formatted for distribution within an online platform (SoGoSurvey, Herndon, Virginia, https:// www.sogosurvey.com/).

To assess who delivers asthma and pMDI education, the providers were asked how often they deliver such education themselves and who else besides them provides pMDI education in their practice. The responses to these 2 questions were pooled and categorized into 5 groups: physician or nurse practitioner; medical assistant, physician assistant, registered nurse, licensed vocational nurse, or asthma educator; other; no one; and unsure.

To assess inhaled medication prescribing habits, the providers were asked about the device they most often prescribe and how often they prescribe spacers to several age groups $(0-3,3-6,6-12$, and $>12$ y). To assess educational methods, the providers were asked to select all education modes used for initial pMDI instruction, including verbal, provider demonstration; patient practice of pMDI use with provider feedback; and provision of printed materials, a training video, or a computer-based tutorial. The providers were also asked about the frequency of pMDI technique reassessment both when a patient's asthma is and is not well controlled and about the mode used to evaluate technique.

To assess attitudes about pMDI education, the providers rated their level of agreement with several statements, such as "proper inhaler technique plays a significant role in asthma control," and "given my current time and resources, I am able to regularly assess patients' inhaler technique in standard office visits." The 5-point Likert scale answer choices ranged from 1 [strongly disagree] to 5 [strongly agree].

To assess potential barriers to $\mathrm{pMDI}$ education, the providers were asked to identify the most significant barriers to regular assessment of patient's inhaler technique. Answer choices included time, access to inhaler devices, health providers' knowledge of proper inhaler technique, language differences between the provider and patients, the lack of interest of patients in proper inhaler technique, no significant barriers, and a free-response option. The providers were queried on how they learned the proper steps in inhaler use and how they learned to teach these steps to patients. Answer choices consisted of personal experience, personal research, training during medical or nursing school, training during residency, a resource (eg, pamphlet, video) at their practice, another health-care provider, an organized training, "I have not learned," or "other," with an option for free response. The providers were also asked to rate their confidence in their ability to teach the proper technique and their perception of the effectiveness of their instruction. The 5 answer options ranged from "not at all confident/effective" to "completely confident/effective." We included a final free-response question that asked providers to identify, assuming no barriers, what methods or resources would be most effective to teach patients the proper inhaler technique.

Demographic information consisted of sex, type of medical training (Doctor of Medicine [MD], Doctor of Osteopathic Medicine [DO], Nurse Practitioner [NP], Registered Nurse [RN], Physician Assistant [PA], Medical Assistant [MA], or Licensed Vocational Nurse [LVN]), years in practice $(0-4,5-9,10-14$, or $\geq 15)$, and the providers' personal experience with asthma, including whether they have active asthma or have a child with active asthma and whether they or their child uses an inhaler with a spacer. The providers were asked to identify their practice setting (independent private practice, large single- or multiple-specialty group private practice, Kaiser Permanente associated, university associated, federally qualified health center, military associated, or other) and to describe their patient population (percentages with asthma, speak Spanish, or receive Medicaid insurance). Use of a Spanish translator was also queried (1 [never] to 5 [always]). 


\section{pMDI Instruction in Pediatric Primary Care}

Table 1. Provider Demographics

\begin{tabular}{lc}
\hline \hline \multicolumn{1}{c}{ Demographics } & $n(\%)$ \\
\hline Sex & \\
Women & $48(77)$ \\
Men & $14(23)$ \\
Practice setting & \\
Private practice: large single- or multi-specialty group & $38(61)$ \\
Federally qualified health center & $12(19)$ \\
Private practice: independent & $6(10)$ \\
University associated & $6(10)$ \\
Medical training & \\
Doctor of medicine & $52(84)$ \\
Nurse practitioner & $7(11)$ \\
Doctor of osteopathic medicine & $2(3)$ \\
Physician assistant & $1(2)$ \\
In practice & \\
$0-4$ y & $12(19)$ \\
$5-9$ y & $15(24)$ \\
10-14 y & $5(8)$ \\
$\geq 15$ y & $30(48)$ \\
\hline
\end{tabular}

\section{Data Analysis}

Standard summary statistics (means and frequencies) were used to describe patient and provider demographics, and provider practices and attitudes about pMDI education. Five-point Likert scale responses were categorized into 3 groups, combining groups "1 and 2" and " 4 and 5" to create a 3-point scale. Free-response answers were reviewed and summarized into recurrent themes. We (DS, KER, ESF) reviewed the responses and achieved consensus on categorizations and themes.

\section{Results}

The response rate was 28\% (62/223); 54 physicians and 8 nurse practitioner/physician assistants participated. Provider demographics are shown in Table 1 . The majority of the responding providers, $71 \%$ (44/62), were associated with private practice groups of varying sizes, whereas $19 \%$ $(12 / 62)$ were from federally qualified health centers, and $10 \%(6 / 62)$ were from university-associated practices. All the respondents identified at least a $5 \%$ prevalence of asthma in their practice, and $35 \%(22 / 62)$ estimated that $\geq 15 \%$ of their own patients had asthma. More than one third of the providers reported either having active asthma themselves or a child with active asthma. The majority of the providers, 58\% (36/62), reported prescribing pMDIs to patients with asthma ages $0-3 \mathrm{y}$, whereas $>93 \%$ prescribed pMDIs to patients with asthma in each of the remaining age groups.

\section{Initial Instruction}

Approximately a third of respondents reported "always" delivering education about inhaler use. Physicians and nurse practitioners provided pMDI education most often (53\%), followed by physician assistants, medical assistants, nurses, and asthma educators (27\%). Several providers (11\%) were unsure if anyone other than themselves provided inhaler education. Nearly all the respondents, 95\% (59/62), agreed that proper inhaler technique plays a significant role in asthma control, and 97\% (60/62) agreed that patients should receive formal instruction in inhaler use when first prescribed a new inhaler. When instructing a patient in pMDI use for the first time, $81 \%$ (50/62) reported giving a verbal description of proper technique and 52\% (32/62) demonstrated the use of the inhaler. Only 10\% (6/62) reported having the patient practice the use of the inhaler in the office and receive feedback (Fig. 1). Overall, 65\% (40/62) reported that they were mostly or completely confident in their ability to teach the pMDI technique, and 48\% (30/62) thought their instruction was mostly or completely effective.

\section{Repeated Instruction}

Most providers, $85 \%$ (53/62), agreed that patients require periodic reassessment of their inhaler technique to ensure maintenance of pMDI skill over time. However, only $19 \%$ of the respondents (12/62) reported "always" reassessing pMDI technique when a patient's asthma is not well controlled. When reassessing technique at follow-up visits, 76\% (47/62) reported asking patients for a verbal description of how they used their inhaler, and $42 \%$ (26/62) reported asking the patient to demonstrate the use of his or her inhaler (Fig. 2).

\section{Barriers and Solutions}

Overall, only $32 \%$ of the respondents (20/62) thought that the typical patient education in their setting was adequate to ensure proper inhaler use. Just 23\% (14/62) agreed that they were regularly able to assess patients' technique in standard office visits. Commonly cited barriers to inhaler education included time, access to a demo inhaler, lack of patient interest, and language differences between providers and patients (Fig. 3). Frequently identified solutions included providing physicians access to demo inhalers and creating computer tutorials to assist patients in learning proper pMDI technique.

\section{Discussion}

In our survey of 62 pediatric out-patient providers in San Diego County, we found a notable disparity between provider attitudes and actual practices pertaining to pMDI technique instruction and assessment. Almost all the providers agreed that formal pMDI instruction was important for asthma control. However, most providers in our study 


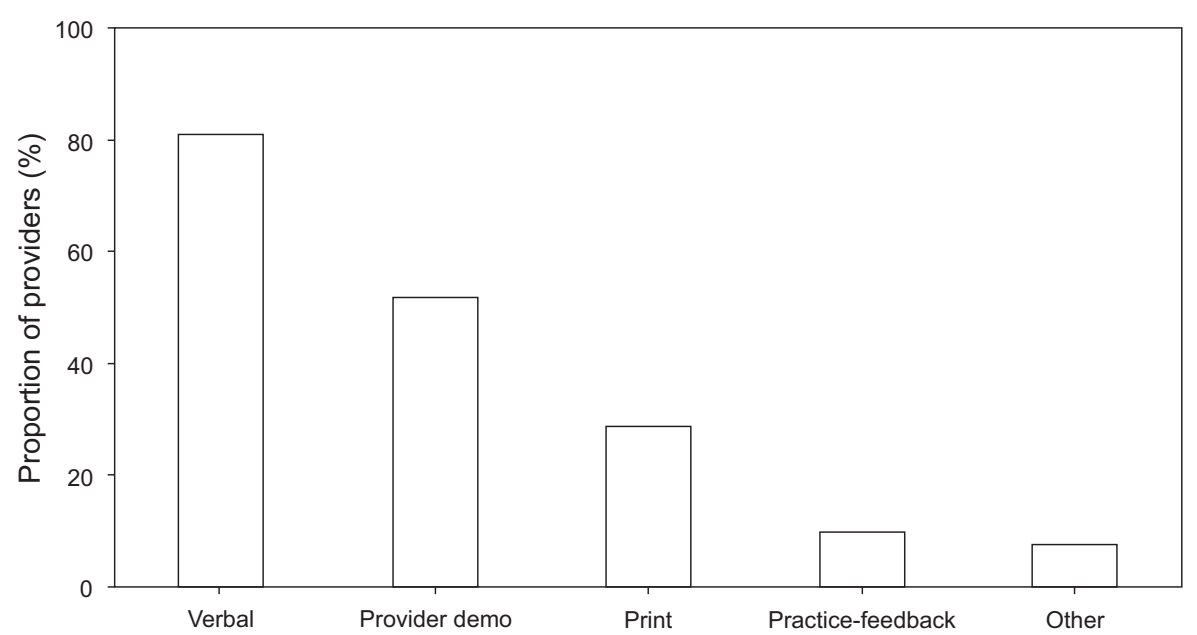

Fig. 1. Methods of initial pressurized metered-dose $(\mathrm{pMDI})$ inhaler use instruction. Verbal = verbal instruction; provider demo $=$ health provider demonstrates pMDI use; print = printed material provided; practice-feedback = patient practices pMDI use and receives feedback.

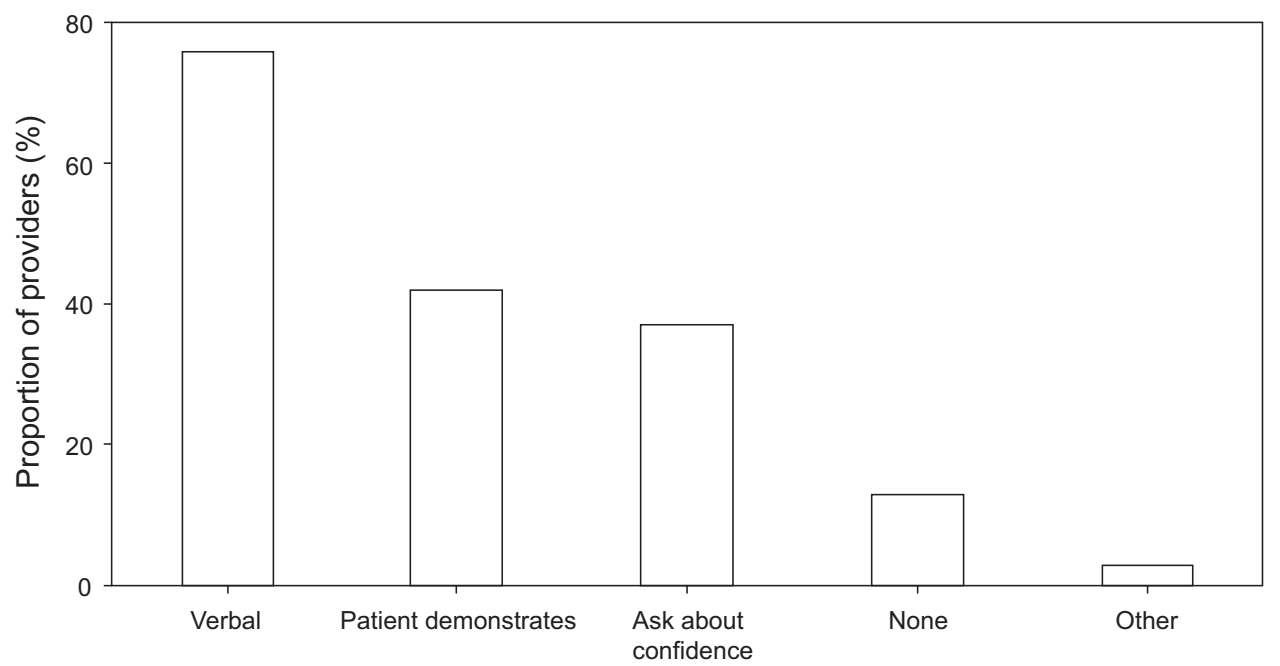

Fig. 2. Methods of pressurized metered-dose inhaler (pMDI) technique reassessment. Verbal = provider asks the patient for a verbal description of pMDI technique; patient demonstrates = provider asks the patient to demonstrate pMDI use; ask about confidence = provider asks the patient about the patient's confidence in pMDI use; none = provider does not regularly reassess pMDI technique.

did not adhere to the NHLBI guidelines ${ }^{12}$ that recommend demonstrating inhaler technique and having patients practice and receive feedback. Previous studies show that subjects who were taught in this manner were more likely than others to demonstrate proper technique at follow-up visits. ${ }^{8,9,11}$ In our study, just over half of the providers reported demonstrating inhaler use to patients when first prescribing pMDIs, and only $10 \%$ reported having patients practice pMDI use and receive feedback. This suboptimal initial education may contribute to the elevated rate of inadequate pMDI technique that has been identified previously. ${ }^{3}$ Respiratory therapists could help fill this education gap because they have been successful in assessing and teaching this technique to patients and families. ${ }^{15-17}$ Although involvement of respiratory therapists in ambu- latory pediatric practices is not new, it is most often reported in subspecialty clinic settings. ${ }^{15,16}$ Respiratory therapists may not have been a consideration by respondents due to a lack of exposure to this kind of integrated care team model in the primary care setting. Extending respiratory therapists' expertise to the general ambulatory pediatric sites could also improve the quality of instruction of medication administration.

The providers also did not adhere to NHLBI guidelines ${ }^{12}$ regarding assessment of inhaler technique at follow-up visits. Despite agreement that periodic reassessment is needed to ensure the proper pMDI technique, $<20 \%$ of the providers reported always reassessing the technique even when asthma was poorly controlled. When they did reassess technique, fewer than half reported having the 


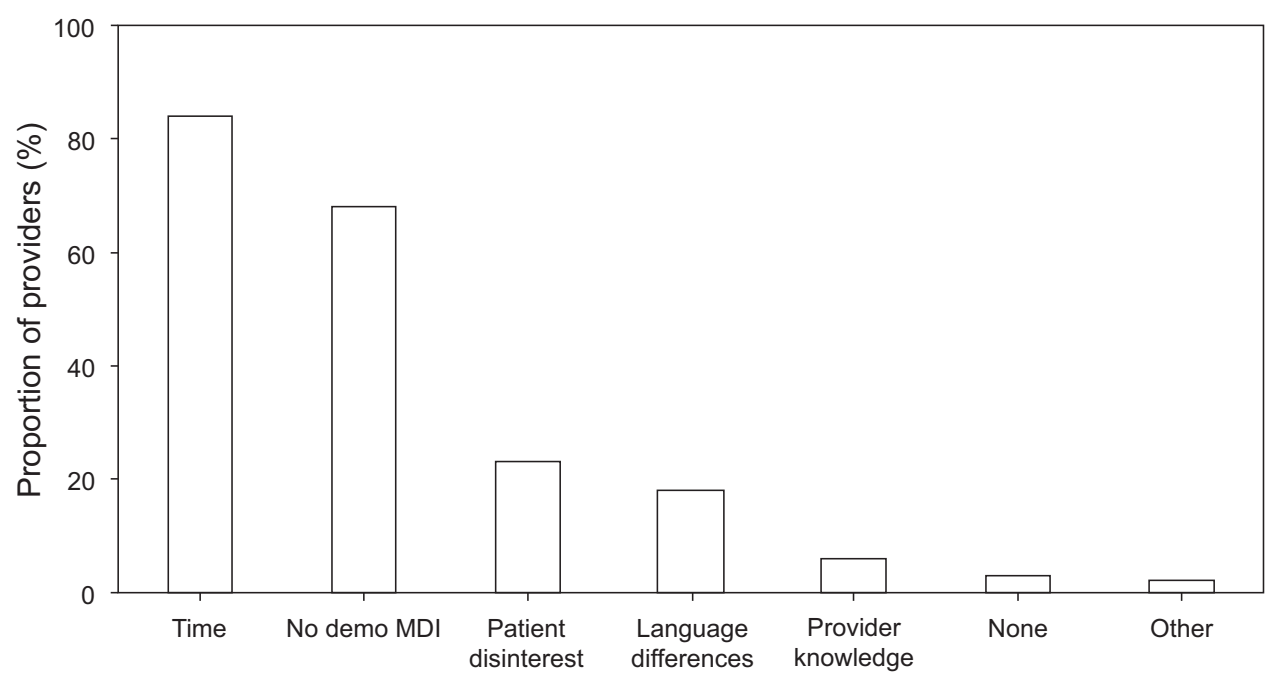

Fig. 3. Barriers to assessment of pressurized metered-dose inhaler (pMDI) technique. No demo pMDI = lack of access to a pMDI for demonstration; patient disinterest $=$ lack of patient interest in learning proper inhaler technique; language differences $=$ language differences between provider and patient; provider knowledge = lack of provider knowledge of pMDI technique.

patient demonstrate the proper use of his or her inhaler. This low rate of reassessment represents a missed opportunity to improve patients' inhaler use skills because repeated instruction and patient demonstration of inhaler use have both been associated with improved technique., ${ }^{5-11}$ Without reassessing patient skills, many providers may not recognize how pMDI use may play into their patients' adherence to medication regimens. Although further study is needed to assess the impact of improved inhaler technique on clinical outcomes, ${ }^{18}$ interventions to enhance pMDI instruction and skill reassessment have the potential to facilitate better disease management through improved pMDI use.

Most providers in this study recognized that patient education in their setting was inadequate to ensure proper inhaler use. Barriers to assessing patient technique included time, access to demonstration inhalers, patient disinterest, and language differences between patients and providers. The providers suggested solutions, including access to sample pMDIs and computer tutorials. Analysis of these data supports the work of Reznik et al ${ }^{14}$ that similarly identified time and access to sample pMDIs in the clinic as significant challenges. Providers in that study, as well as ours, suggested that having a nurse or health educator deliver asthma education could alleviate time constraints. ${ }^{14} \mathrm{~A}$ logical extension of this idea would be to embrace a true interdisciplinary practice or collaborative care model in which respiratory therapists perform this educator role. Using computer tutorials could similarly streamline pMDI use education and would be useful in addition to or integrated with in-person education. Such tutorials used in isolation, however, could not accomplish the key step of having patients practice pMDI use and receive feedback from a skilled provider. Supplying clinics with demo pMDIs would support the ability to regularly perform pMDI instruction. Further studies are needed to explore the impact of perceived patient disinterest and language differences on pMDI use education.

The above-mentioned interventions could also facilitate a complementary approach to pMDI education supported by theories of motor learning. Proper use of a pMDI involves motor skills that require coordinating several movement tasks with an appropriate order and timing. Although motor skills can be mastered effectively during brief sessions spaced over days, ${ }^{19,20}$ ongoing skill use and training are needed to maintain proficiency over time. ${ }^{6,7}$ Therefore, pMDI use could be most efficiently learned through brief but consistent practice in the days after initial instruction until the skill is mastered, coupled with reassessment and retraining to assure that the skill is retained. An up-front investment in the time and resources for this strategy could have longterm implications for improved asthma management.

Despite our key findings of areas for improvement in the teaching and assessment of pMDI use, there were a few limitations to consider. Our responses were obtained via an online survey. Although this method was chosen to decrease participant burden and increase the response rate across a range of practice settings, it may instead have resulted in fewer respondents. Survey methodology can also limit the range of responses that are captured. Telephone surveys, key informant interviews, or focus groups would have allowed for a greater range of responses and exploration of barriers about pMDI instruction. Survey research has limitations, such as data entry errors, reliance on memory, and dependence on respondents' correct interpretation of the questions. There may also have been a 


\section{pMDi Instruction in Pediatric Primary Care}

response bias because the participants who have a vested interest in this topic are more likely to complete the survey. The self-report design also raised the possibility of discrepancies between provider perceptions and actual practices within the clinics. Unfortunately, we were unable to objectively assess the competency of the providers in their pMDI technique. Also, the relatively small sample size and low response rate limited the generalizability of our data and precluded potential subgroup analysis, including variation in pMDI use education by practice type. For this reason, a subgroup analysis that could show potential differences by professional group (separating physicians and nurse practitioners) and the duration of practice was not performed. However, we were able to include different practice setting models than in previous studies and offered a greater variety of potential solutions that could improve the effectiveness of pMDI use instruction in the out-patient setting.

\section{Conclusions}

This study of ambulatory primary care pediatric sites supported previous studies' descriptions of guideline-topractice gaps in pMDI use education and offered potential targets for quality improvement initiatives in the out-patient setting. Consideration should be given to extending the role of respiratory therapists to the general pediatric ambulatory practice setting to fill this gap. This study adds to the field for childhood asthma by offering ambulatory generalist perspectives related to pMDI use education at both first use and subsequently, and identifying generalistreported common barriers and solutions, such as using nurse educators, sample pMDIs, and computer tutorials. Future research should further investigate the effect of improved pMDI technique on clinical outcomes and the potential for several education sessions spaced over days to promote stable mastery of pMDI use skills.

\section{REFERENCES}

1. Centers for Disease Control and Prevention. 2015 National Health Interview Survey (NHIS) Data, Table 3-1. https://www.cdc.gov/ asthma/nhis/2015/table3-1.htm. Accessed March 7, 2018.

2. O'Callaghan C. Delivery systems: the science. Pediatr Pulmonol Suppl 1997;15:51-54.

3. Fink JB, Rubin BK. Problems with inhaler use: a call for improved clinician and patient education. Respir Care 2005;50(10):1360-1374; discussion 1374-1375.

4. Sanchis J, Gich I, Pedersen S; Aerosol Drug Management Improvement Team (ADMIT). Systematic Review of Errors in Inhaler Use:
Has Patient Technique Improved Over Time? Chest 2016;150(2): 394-406.

5. Melani AS, Bonavia M, Cilenti V, Cinti C, Lodi M, Martucci P, et al.; Gruppo Educazionale Associazione Italiana Pneumologi Ospedalieri. Inhaler mishandling remains common in real life and is associated with reduced disease control. Respir Med 2011;105(6):930-938.

6. Klijn SL, Hiligsmann M, Evers SMAA, Román-Rodríguez M, van der Molen T, van Boven JFM. Effectiveness and success factors of educational inhaler technique interventions in asthma \& COPD patients: a systematic review. NPJ Prim Care Respir Med 2017;27(1):24.

7. Munzenberger PJ, Thomas R, Bahrainwala A. Retention by children of device technique for inhaled asthma drugs between visits. J Asthma 2007;44(9):769-773.

8. Bosnic-Anticevich SZ, Sinha H, So S, Reddel HK. Metered-dose inhaler technique: the effect of two educational interventions delivered in community pharmacy over time. J Asthma 2010;47(3):251256.

9. Kamps AW, Brand PL, Roorda RJ. Determinants of correct inhalation technique in children attending a hospital-based asthma clinic. Acta Paediatr 2002;91(2):159-163.

10. Kamps AW, van Ewijk B, Roorda RJ, Brand PL. Poor inhalation technique, even after inhalation instructions, in children with asthma. Pediatr Pulmonol 2000;29(1):39-42.

11. Sleath B, Carpenter DM, Ayala GX, Williams D, Davis S, Tudor G, et al. Communication during pediatric asthma visits and child asthma medication device technique 1 month later. J Asthma 2012;49(9): 918-925.

12. National Heart, Lung, and Blood Institute. Expert Panel 3 (EPR 3). Guidelines for the Diagnosis and Management of Asthma 2007. https://www.nhlbi.nih.gov/health-topics/guidelines-for-diagnosismanagement-of-asthma. Accessed March 7, 2018.

13. Sleath B, Ayala GX, Gillette C, Williams D, Davis S, Tudor G, et al. Provider demonstration and assessment of child device technique during pediatric asthma visits. Pediatrics 2011;127(4):642-648.

14. Reznik M, Jaramillo Y, Wylie-Rosett J. Demonstrating and assessing metered-dose inhaler-spacer technique: pediatric care providers' selfreported practices and perceived barriers. Clin Pediatr (Phila) 2014; 53(3):270-276.

15. Kallstrom TJ, Myers TR. Asthma disease management and the respiratory therapist. Respir Care 2008;53(6):770-776; discussion 777.

16. McClain BL, Ivy ZK, Bryant V, Rodeghier M, DeBaun MR. Improved Guideline Adherence With Integrated Sickle Cell Disease and Asthma Care. Am J Prev Med 2016;51(1 Suppl 1):S62-68.

17. Minai BA, Martin JE, Cohn RC. Results of a Physician and Respiratory Therapist Collaborative Effort to Improve Long-Term Metered-Dose Inhaler Technique in a Pediatric Asthma Clinic. Respir Care 2004;49(6):600-605.

18. Normansell R, Kew K, Stovold E, Mathioudakis AG, Dennett E. Interventions to improve inhaler technique and adherence to inhaled corticosteroids in children with asthma. Paediatr Respir Rev 2017; 23:53-55.

19. Savion-Lemieux T, Penhune VB. The effects of practice and delay on motor skill learning and retention. Exp Brain Res 2005;161(4): 423-431.

20. Smith CD, Scarf D. Spacing Repetitions Over Long Timescales: A Review and a Reconsolidation Explanation. Front Psychol 2017;8:962. 